\title{
Impacts of low molecular weight heparin on bone healing and osseointegration
}

\author{
Yu Mori ${ }^{1}\left[\right.$ Toshimi Aizawa $^{1}$
}

Received: 3 November 2021 / Accepted: 14 November 2021 / Published online: 14 January 2022

(c) The Japanese Society Bone and Mineral Research 2021

\section{To the Editor}

We read with interest the article by Li et al. titled "Impaired bone healing by enoxaparin via inhibiting the differentiation of bone marrow mesenchymal stem cells towards osteoblasts" [1]. It has been previously reported that heparin and low-molecular-weight heparin are associated with the suppression of bone formation and enhancement of bone resorption in experimental models [2]. The risks of osteoporosis and fragility fractures have been discussed in patients using heparin and low-molecular-weight heparin for anticoagulation [3]. Enoxaparin is widely used in the postoperative period of arthroplasty to prevent thrombosis and embolism. We believe that it would be interesting for practicing orthopedic surgeons to learn about the possible impacts of enoxaparin on osseointegration of metal surfaces after cementless total hip and knee arthroplasties. However, the use of enoxaparin for the prevention of thrombosis is limited to approximately 10 days after surgery. Considering its short-term use, the extent of its influence on the differentiation of bone marrow mesenchymal stem cells into osteoblasts is debatable. In addition, since the inhibition of bone formation was evaluated only by computed tomography in this study, we believe that a more detailed understanding of enoxaparin's inhibition of bone formation will be obtained by performing histological and bone strength studies using mechanical tests.

In clinical settings, bone defects are usually treated using autologous bone grafting or bone graft substitutes. We had created bilateral cortical bone defects with a diameter of $3 \mathrm{~mm}$ in rat femur, simulating bone defects that cannot be repaired spontaneously, and investigated the mechanism of bone repair using octacalcium phosphate composite [4]. It would be more clinically meaningful to evaluate the effect of

Yu Mori

yu-mori@med.tohoku.ac.jp

1 Department of Orthopaedic Surgery, Tohoku University Graduate School of Medicine, 1-1 Seiryo-machi, Aoba-ku, Sendai, Miyagi 980-8574, Japan enoxaparin on bone healing using autologous bone grafting or bone graft substitutes.

Low-molecular-weight heparin is commonly used for the prevention of thrombosis after joint replacement surgery, and clinicians are interested in the impact of enoxaparin on osseointegration of the metal surface of cementless prostheses. Aiming to enhance the osteoinductive ability of cementless prostheses, we had investigated the effect of anodic oxidation of titanium alloy on osseointegration [5]. We believe that this research will more reliable when the impact of enoxaparin on osseointegration on the metal surface will be evaluated by a pull-out test after implanting the metal materials.

\section{References}

1. Li Y, Liu L, Li S, Sun H, Zhang Y, Duan Z, Wang D (2021) Impaired bone healing by enoxaparin via inhibiting the differentiation of bone marrow mesenchymal stem cells towards osteoblasts. J Bone Miner Metab. https://doi.org/10.1007/s00774-021-01268-5

2. Muir JM, Hirsh J, Weitz JI, Andrew M, Young E, Shaughnessy SG (1997) A histomorphometric comparison of the effects of heparin and low-molecular-weight heparin on cancellous bone in rats. Blood 89:3236-3242. https://doi.org/10.1182/blood.V89.9.3236

3. Signorelli SS, Scuto S, Marino E, Giusti M, Xourafa A, Gaudio A (2019) Anticoagulants and osteoporosis. Int J Mol Sci. https://doi. org/10.3390/ijms20215275

4. Oizumi I, Hamai R, Shiwaku Y, Mori Y, Anada T, Baba K, Miyatake N, Hamada S, Tsuchiya K, Nishimura SN, Itoi E, Suzuki O (2021) Impact of simultaneous hydrolysis of OCP and PLGA on bone induction of a PLGA-OCP composite scaffold in a rat femoral defect. Acta Biomater 124:358-373. https://doi.org/10.1016/j. actbio.2021.01.048

5. Kunii T, Mori Y, Tanaka H, Kogure A, Kamimura M, Mori N, Hanada S, Masahashi N, Itoi E (2019) Improved osseointegration of a TiNbSn alloy with a low Young's modulus treated with anodic oxidation. Sci Rep 9:13985. https://doi.org/10.1038/ s41598-019-50581-7

Publisher's Note Springer Nature remains neutral with regard to jurisdictional claims in published maps and institutional affiliations. 